\title{
A Stiffness Variable Passive Compliance Device with Reconfigurable Elastic Inner Skeleton and Origami Shell
}

\author{
Zhuang Zhang ${ }^{1,2}$, Genliang Chen ${ }^{1,2^{*}}$, Weicheng Fan' ${ }^{1}$, Wei Yan ${ }^{1}$, Lingyu Kong ${ }^{3}$ and Hao Wang ${ }^{1,2}$
}

\begin{abstract}
Devices with variable stiffness are drawing more and more attention with the growing interests of human-robot interaction, wearable robotics, rehabilitation robotics, etc. In this paper, the authors report on the design, analysis and experiments of a stiffness variable passive compliant device whose structure is a combination of a reconfigurable elastic inner skeleton and an origami shell. The main concept of the reconfigurable skeleton is to have two elastic trapezoid four-bar linkages arranged in orthogonal. The stiffness variation generates from the passive deflection of the elastic limbs and is realized by actively switching the arrangement of the leaf springs and the passive joints in a fast, simple and straightforward manner. The kinetostatics and the compliance of the device are analyzed based on an efficient approach to the large deflection problem of the elastic links. A prototype is fabricated to conduct experiments for the assessment of the proposed concept. The results show that the prototype possesses relatively low stiffness under the compliant status and high stiffness under the stiff status with a status switching speed around $80 \mathrm{~ms}$.
\end{abstract}

Keywords: Variable stiffness, Passive compliance device, Reconfigurable skeleton, Origami shell

\section{Introduction}

Human-Robot Interaction is one of the most challenging and popular research topics in robotics [1]. Meanwhile, for an effective interaction, the safety of human is of importance. Cartesian compliance [2] can ensure the safety of both robot manipulators and human when contact force arises from position misalignment or hard collision [3]. In general, there are mainly two methods to protect both the manipulators and human from being damaged by unexpected interaction, namely the active and the passive compliance [4].

The active compliance is to manipulate robot with totally stiff actuators and obtain compliance by means of control [5]. Such kind of compliance requires force sensing units to provide force information to the controller [6]. However, if there is something wrong with

\footnotetext{
*Correspondence: leungchan@sjtu.edu.cn

${ }^{1}$ Shanghai Key Laboratory of Digital Manufacture for Thin-walled

Structures, Shanghai Jiao Tong University, Shanghai 200240, China

Full list of author information is available at the end of the article
}

the sensors or the controllers, the manipulator will thoroughly lose its compliance and will not be able to ensure safety [7]. Another way to attain compliance is integrating flexible structures into the manipulators, which is referred to as passive compliance [8]. Such kind of compliance provides more reliable protection which is irrelevant to the control algorithm [9], thanks to its inherent flexible structure. The passive compliance can be obtained by setting compliant device between the manipulator and environment [10-14] or integrating the compliant joints inside the manipulator [15-18]. However, the integration of compliant elements will lead to degradation of position accuracy. Besides, the structural compliance also brings challenges in resisting disturbances or maintaining shapes under intense inertia, which will limit the performance of the manipulators under high acceleration [19].

To this end, various designs of passive variable stiffness joints/devices have been proposed. One of them is structure controlled stiffness (SCS) that means achieving variations through changing the effective structure of 
the spring. The core advantage of SCS is that completely stiff setting is possible to realize. As a consequence, SCS is more appropriate than other designs when precise positioning or high acceleration is needed. Changing the moment of inertia is one of the feasible methods to vary the effective structure. Kawamura et al. [20] used vacuum to press some layered sheets together. The moment of inertia changes along with the variation of the effective cross-sectional area. Such method of stiffness variation has been widely used in soft grippers and manipulators due to its simple structure and control strategy [19, 21-23]. Another way to change the effective structure is controlling the effective length of the spring. Choi et al. [18] designed a variable stiffness joint which consisted of four-bar linkages, leaf springs and two identical actuators. Controlling the four-bar linkages on the two sides identically, the effective lengths of the springs could be changed. Tao et al. [24] proposed a variable stiffness joint with similar principle. Only one leaf spring was used and the effective length was changed by rollers and a screw. Bi et al. [25] presented a concept of parallel-assembledfolded serial leaf springs. The lengths of the springs were also controlled by rollers and a ball screw.

Among the existing designs of SCS that can provide tunable passive compliance, most of them were compliant joints. The stiffness varies along with the tuning of the length or thickness of the spring. Thus, the elements to control the effective structure of the springs are indispensable, which makes the structure of the joints complicated and hard to integrate inside the robots under some specific applications. Moreover, most of the designs utilize electrical motors to control stiffness. The motors generally need to apply torque to keep a constant stiffness, which is energy inefficient. In addition, the motors introduce relatively high weights and is not suitable for extreme environments.

To alleviate the above shortcomings, in this paper, a novel design of a stiffness variable passive compliance device with combined structure of reconfigurable elastic inner skeleton and origami shell is proposed. The proposed device can be used as an end-of-arm tool with two different modes generated from changing the arrangement of the elastic links and the passive joints. Apart from providing passive compliance, the device can switch to the stiff status for applications with high acceleration/ deceleration or precise positioning. With a Si-Mo (single input multiple output) pneumatic actuation system, the four limbs of the inner skeleton can switch their modes simultaneously and the stiffness of the device can be changed in a fast, simple and straightforward manner. No electrical elements needs to be mounted on the device, which means the device has potential applications in some extreme environments. The kinetostatics and the compliance of the device are analyzed based on an efficient approach to large deflection problems [26, 27]. A prototype was built, on which experimental assessments of the stiffness changing capabilities were conducted.

The paper is arranged as follows: Section 2 introduces the concept of the stiffness variation of the device. Section 3 provides a detailed description of the mechanical design and the fabrication method. The theoretical model and the compliance of the device are analyzed in Section 4, followed in Section 5 by experimental assessments on the fabricated prototype. Finally, conclusions are drawn in Section 6.

\section{Concept of the Stiffness Variation}

The structure of the proposed passive compliance device with variable stiffness can be divided into three parts: the reconfigurable elastic inner skeleton, the origami shell, and the Si-Mo pneumatic actuation system, as shown in Figure 1. Among these parts, the reconfigurable elastic inner skeleton is the core component from which the passive compliance and the variable stiffness generate.

\subsection{Reconfigurable Elastic Inner Skeleton}

The main concept of the reconfigurable [28-30] elastic inner skeleton is to have two trapezoid four-bar linkages arranged in orthogonal. As shown in Figure 1, each trapezoid consists of two rigid limbs $(\mathrm{AD} / \mathrm{ad}, \mathrm{BC} / \mathrm{bc})$ and two elastic limbs $(\mathrm{AB} / \mathrm{ab}, \mathrm{DC} / \mathrm{dc})$. The rigid limbs are mounting side and tool side of the device, respectively. There are four elastic limbs in the device while each elastic limb consists of two universal joints and a leaf spring. The motion of each trapezoid four-bar linkage is totally passive and the only active motion inside the device is the self-rotation of the leaf springs. Under the actuation of the Si-Mo pneumatic actuation system, the leaf springs of the four elastic limbs are able to rotate $90^{\circ}$ simultaneously. Figure 2 illustrates the statuses of the device before and after $90^{\circ}$ rotation, respectively.

The leaf spring in each elastic limb plays an important role in the stiffness variation. Considering the small deflection beam equation

$$
M=\left(\frac{E I}{L}\right) \times \theta
$$

where $M$ is the bending moment, $E$ is the material modulus, $I$ is the moment of inertia, $L$ is the effective beam length, and $\theta$ is the angle of bending or slope.

In this representation of bending, the term $E I / L$ relates to the bending stiffness of the leaf spring. In order to change the stiffness of the beam, one of the most effective way is to change the parameter $I$ that can be calculated as 


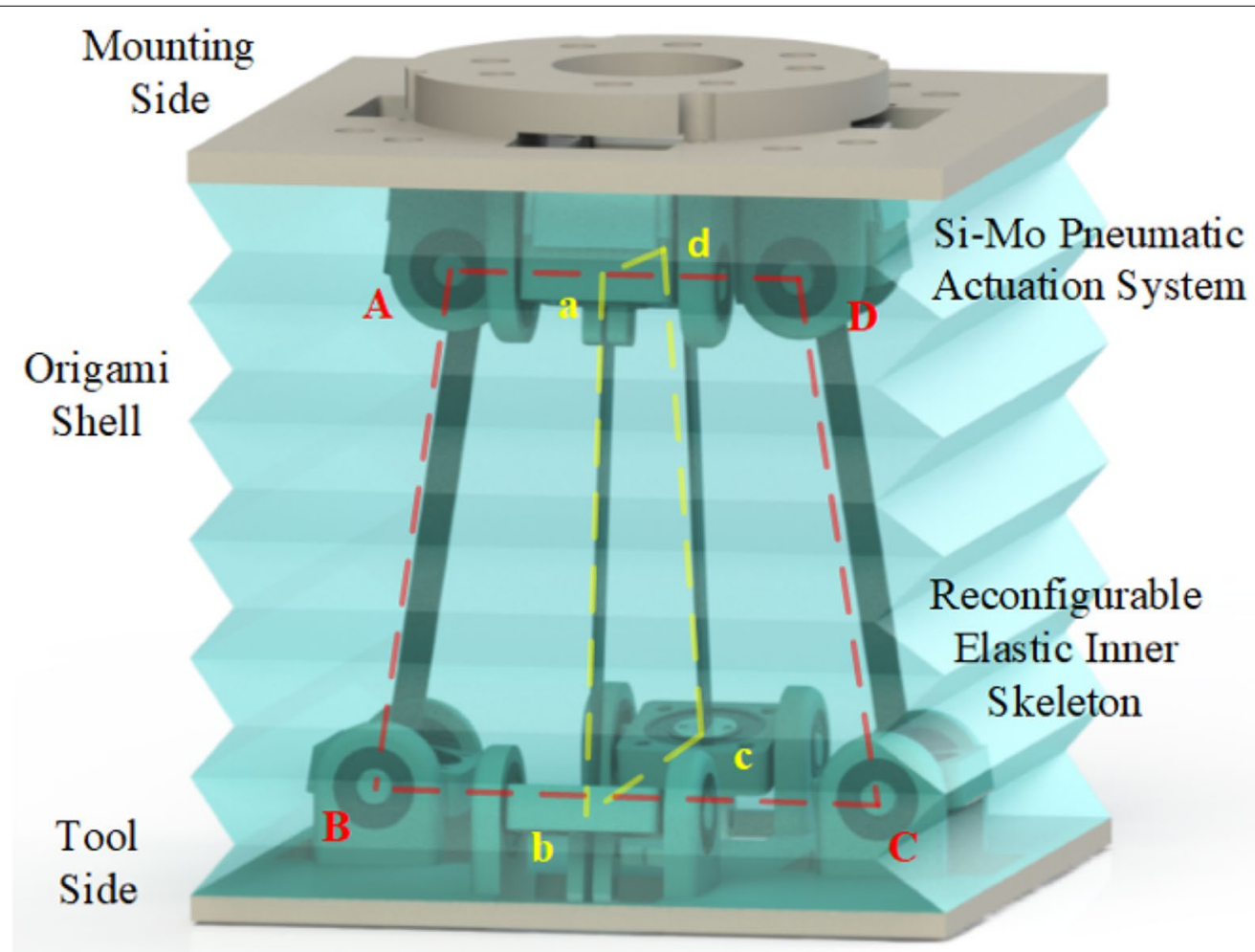

Figure 1 Structure of the proposed variable stiffness device

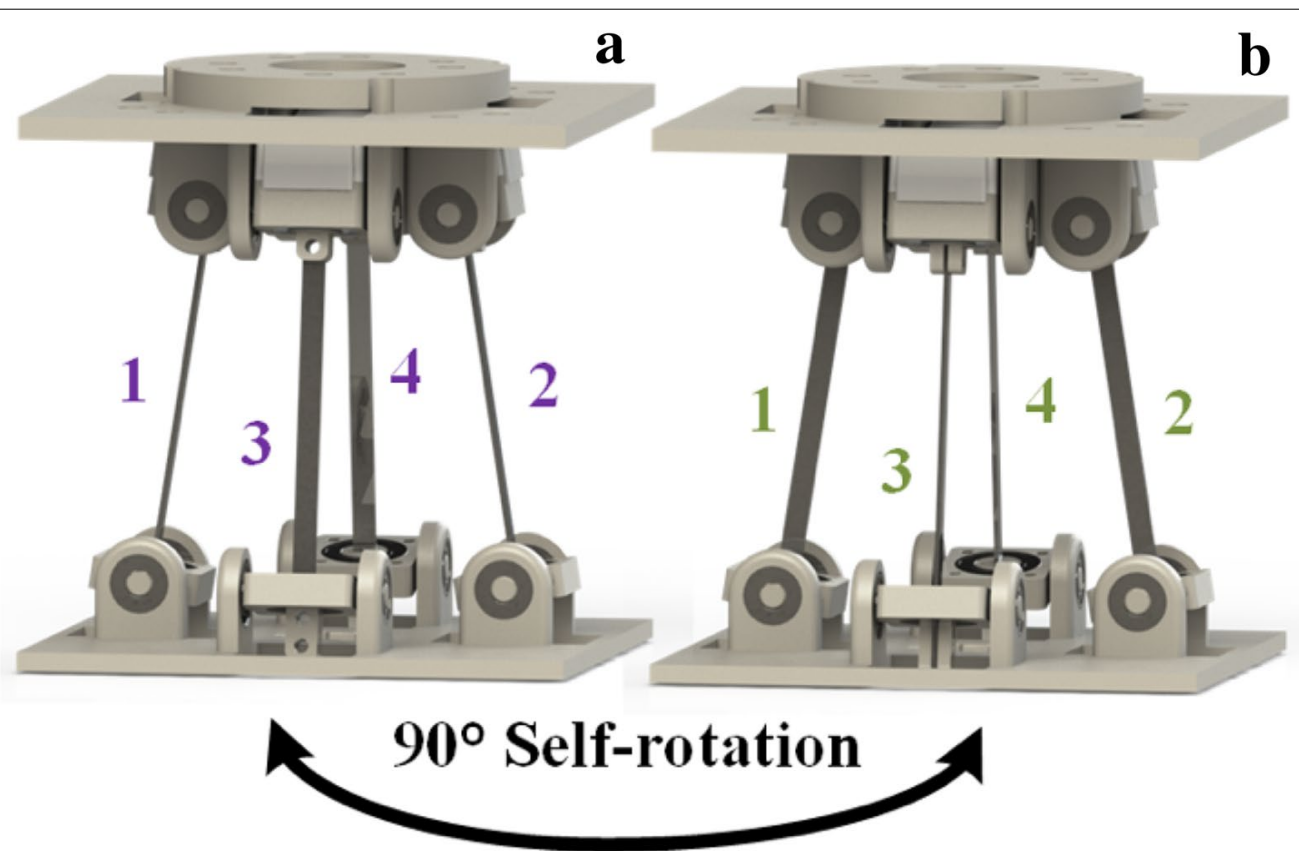

Figure 2 Two different statuses of the inner skeleton 


$$
I=\frac{L_{b} \times L_{h}^{3}}{12},
$$

where the parameter $L_{b}$ denotes the width of the surface perpendicular to the bending direction, and $L_{h}$ denotes the other.

It can be seen that if $L_{b}$ and $L_{h}$ exchange with each other, the moment of inertia will be changed. As to the leaf spring, there is a big difference between its width and its thickness. The moment of inertia differs a lot in the two bending directions, so that the stiffness of the leaf spring in the two directions are totally different. By means of such feature, the leaf springs in the proposed device are designed to have the capacity of $90^{\circ}$ self-rotation. Along with the stiffness variation of the leaf springs on the specific directions, the constraints on the tool side can be changed through combining the elastic springs with the passive rigid joints appropriately.

\subsection{Stiff Status of the Device}

Compared with the width, the thickness of the leaf spring is much smaller. Thus, it's appropriate to regard the spring as totally stiff in the direction perpendicular to the narrow edge. As shown in Figure 2(a), when arranging each pair of the leaf springs 'facing each other', the bending direction of the leaf springs is same with the rotation of the corresponding passive joints in the same elastic limbs. Thus, each four-bar linkage only has the capability of planar movement. However, once there is a motion trend in one of the trapezoid four-bar linkages, the leaf springs and the corresponding joints in the other trapezoid will act totally stiff in that direction due to the orthogonal arrangement of two trapezoids. For example, as shown in Figure 2(a), the trapezoid four-bar linkage consisting of elastic limb 1, limb 2 and two rigid plates (namely trapezoid ABCD in Figure 1) has only one Degree-of-Freedom. However, elastic limb 3 and limb 4 act stiff on such moving direction because the direction is perpendicular to the narrow edges of the leaf springs and also the passive joints. As a result, no movement could be realized except that buckling happens. In such status, the device is suitable for precise positioning and motion with high acceleration/deceleration.

\subsection{Compliant Status of the Device}

As shown in Figure 2(b), the bending direction of each leaf spring is perpendicular to the rotation of the corresponding passive joints in the same elastic limb after $90^{\circ}$ self-rotation from the stiff status shown in Figure 2(a). In such situation, no movement could happen when regarding all the springs as rigid body. However, the bending of the leaf spring is easy to realize, so that the tool side of the device will be able to move. The movement of the device can be regarded as a combination of the planar motion of one four-bar linkage and the bending of the other one. As shown in Figure 3, when one of the four-bar linkages starts moving, the corresponding leaf springs are rigid in the moving direction. The one Degree-of-Freedom planar motion is as thus generated. At the same time, the leaf springs in the other four-bar linkage are easy to be deformed on the same direction. Then, deflections happen in these two springs to adapt the movement. As the compliance generates from the structural deflection, the device can provide passive compliance which is irrelevant to the control algorithm.

\section{Design and Fabrication}

\subsection{Reconfigurable Elastic Inner Skeleton}

The design of the inner skeleton is illustrated in Section 2.1. As to the fabrication of the elastic inner skeleton, the main principles of the material selection are lightweight and easy to obtain. All of the non-standard rigid parts are three-dimensional printed. Slender $\mathrm{Ni}-\mathrm{Ti}$ alloy strips are employed as the elastic leaf springs.

\subsection{Origami Shell}

Apart from the structural passive compliance, the elastic limbs with slender structures also bring shortcomings. Under the situation shown in Figure 2(b), the tool side is easy to be twisted relative to the mounting side due to the low stiffness of the leaf springs. Further, the bare slender metal sheets are not safe enough in manipulation.

To alleviate above shortcomings, a tubular origami shell with Yoshimura pattern [31] is integrated to form an enclosed structure and prevent the device from twist, as shown in Figure 1. As an ancient Chinese and Japanese

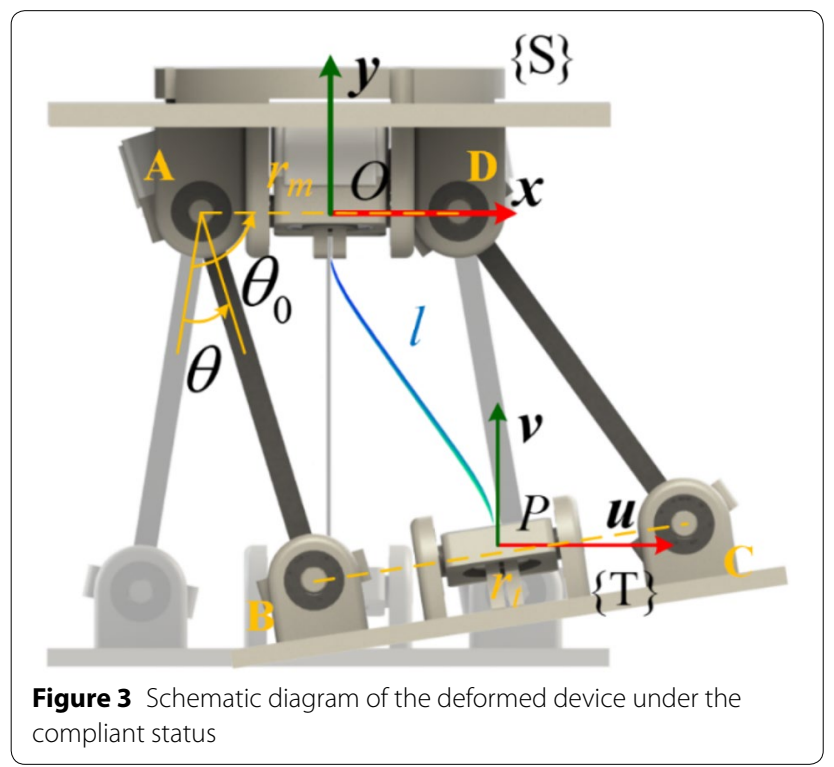


art of paper folding, origami is drawing more and more attention these years in the robotics field and origami robots can be defined as autonomous machines whose morphology and function are created using folding [32-35]. The Yoshimura pattern is a cylindrical folding origami that supports bending and axial folding [36-38]. Another merit of such kind of origami shell is its torsion resistance [39]. In this way, the tendency of twist under the status in Figure 2(b) is avoided without influencing the passive motion of the elastic skeleton. Besides, the slender metal sheets are isolated from the working environment by the compliant origami shell. Hence, the interaction safety is ensured. In addition, the feature of uncontinuously foldable [40] makes the Yoshimura pattern resistant to be axially compressed. Thus, the integration of a pre-compressed origami shell with Yoshimura pattern will provide extension force and prevent the device from buckling.

The base crease pattern of the origami shell as well as the actual images of the machined pattern and the manually folded shell is shown in Figure 4 . The $0.15 \mathrm{~mm}$ polyethylene terephthalate (PET) films are chosen due to their high strength-to-weight ratio, transparency and easy to obtain. The crease pattern is planar designed and machined by a carbon dioxide laser-cutter. It is worth noting that the black solid lines represent mountain creases and the blue dash lines represent valley creases in Figure 4(a). The 2D laser-cut PET film (Figure 4(b)) can be manually folded into a 3D structure following the crease pattern and finally forms a rectangular tube (Figure 4(c)). The rectangular structure is attained from designing four sections in the proposed 2D pattern, which aims to attain similar bending capacities with the reconfigurable elastic inner skeleton under the compliant status.

\subsection{Si-Mo Pneumatic Actuation System}

As discussed above, the variable stiffness of the proposed device generates from the mode switching of the reconfigurable elastic inner skeleton. Hence, in order to actively control the mode of the skeleton, a Si-Mo pneumatic actuation system is designed. The system consists of a pneumatic central line and four sub-actuators, as shown in Figure 5. Each sub-actuator consists of a rigid rotor, a rigid stator and two soft pneumatic chambers. The rigid parts are three-dimensional printed and the pneumatic chambers are made of inelastic air-tight fabric. The two chambers that connect the shank of the rotor and the arc groove of the stator together on the opposite sides will significantly expand and fill the groove when pressurized. In this way, the two chambers antagonistically actuate the rotor to swing inside the stator. The four elastic leaf springs are connected with the four rotors

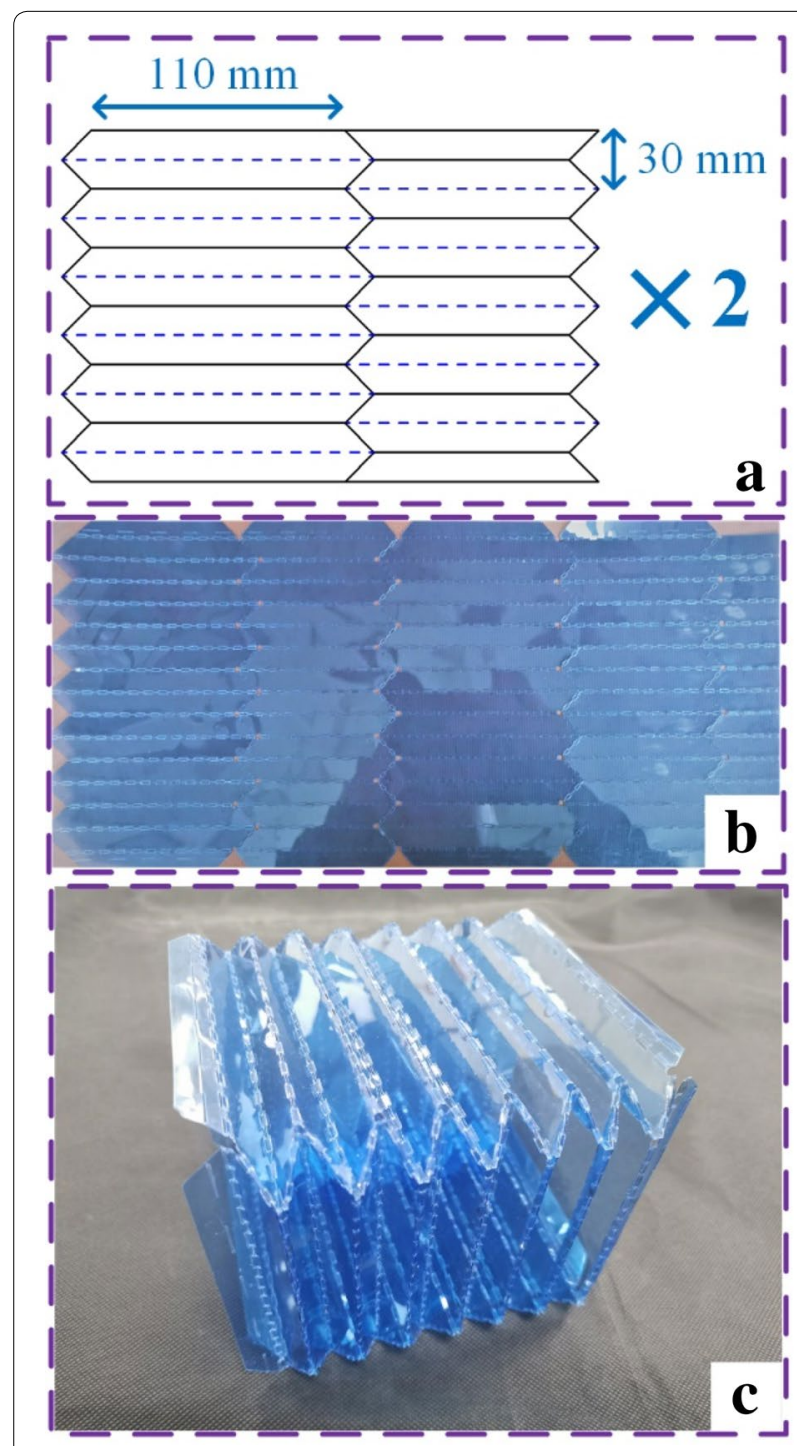

Figure 4 Origami shell: base crease pattern, 2D laser-cut film and folded 3D structure

through bolts and nuts, respectively. As a result, when the rotors rotate under the pneumatic actuation, the leaf springs will correspondingly rotate. In this way, the reconfiguration of the structure is achieved.

The pneumatic central line provides air source to the four sub-actuators that integrated inside the four elastic limbs. A 2-4 way solenoid valve (VQD1121, SMC) is used to switch the inflation between the two chambers of each sub-actuator. Benefiting from the pneumatic actuation, a single pressure input can control four actuators simultaneously, as shown in Figure 5. Moreover, the motion of the limbs has no influence on the transmission of the soft pneumatic pipes when the device is imposed to deform under the compliant status illustrated in Figure 3. Such 


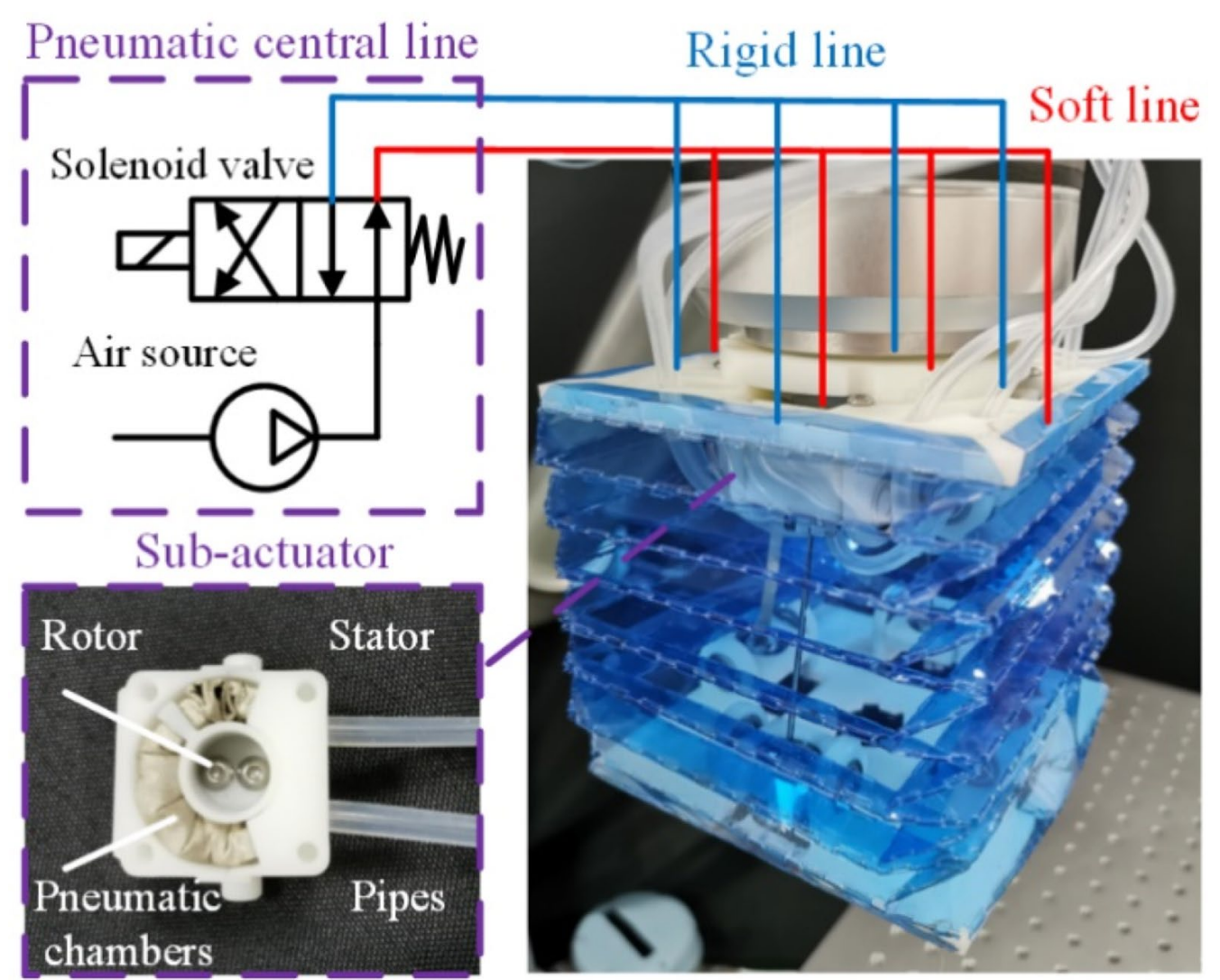

Figure 5 Si-Mo pneumatic actuation system

soft transmission is hard to realize by other transmission methods, such as cable transmission, gear transmission, etc.

Another advantage of such pneumatic actuation is its remote transmission. The control system can be placed far away from the device and the mode switching will not be significantly influenced. As no electrical elements needs to be mounted on the device and the only actuation elements connecting to the device are two soft pipes, the device has potential applications in some special environments, such as underwater, radioactive, low/high temperature, etc.

\section{Modeling and Compliance Analysis}

As discussed in the previous sections, the motion of the tool side can only be produced by external force under the compliant status. The leaf springs will not deform under the stiff status. Hence, the modeling and analysis in this section are based on the compliant status. Due to the orthogonal arrangement, the compliance can be regarded as generating from the combination of two deformed trapezoid four-bar linkages. Each leaf spring acts stiff in the moving direction of the corresponding four-bar linkage and flexible in the moving direction of the other one. Hence, to prove the compliance of the device, it's appropriate to analyze the deflection of the leaf springs and the force they generate in one of the trapezoid linkages. The key issue for the compliance analysis is to analyze the large deflection of the leaf springs. Based on our prior work on the general approach to the large deflection problems of spatial flexible rods [26, 27], the kinetostatics of the leaf springs inside the device can be analyzed. In this way, the compliance of the device under the compliant status can be predicted and the geometric parameter designs for different applications can benefit from the analysis accordingly.

\subsection{Kinematics}

For kinematics modeling, two reference frames, namely the spatial one $\{S\}$ and the tool one $\{T\}$, are constructed at the mounting side and the tool side of the device, respectively. The origin of $\{\mathrm{S}\}$, termed $O$ shown in Figure 3 , locates at the center of the mounting side. The corresponding $x$ and $y$ axes are along the horizontal and the vertical directions, respectively. The origin of $\{\mathrm{T}\}, P$ is referred to as the center of the tool side of the device. 
As indicated above, the four-bar linkage that parallel to the moving direction will keep rigid while the other one deflects to adapt the movement. Hence, the configuration of $\{\mathrm{T}\}$ is uniquely determined according to the motion of the rigid four-bar linkage. Then, the configuration of the tool side can be obtained by solving the following equations: joints associated with the segments' bending and torsion effects will be taken into account in the hyper-redundant mechanism. Using product-of-exponential formula for forward kinematics [43], the configuration of the approximated hyper-redundant mechanism's tip frame $\mathbf{g}_{l t}$ and the balance of the elastic deflection can then be defined as

$$
\left\{\begin{array}{l}
x=-\frac{r_{m}}{2}+\frac{r_{t}}{2} \cos \phi+l \cos \left(\theta_{0}-\theta\right), \\
y=\frac{r_{t}}{2} \sin \phi-l \sin \left(\theta_{0}-\theta\right), \\
\left(-r_{m}+r_{t} \cos \phi+l \cos \left(\theta_{0}-\theta\right)\right)^{2}+\left(r_{t} \sin \phi-l \sin \left(\theta_{0}-\theta\right)\right)^{2}=l^{2},
\end{array}\right.
$$

where $x, y, \phi$ correspond to the horizontal position, the vertical position and the rotation of the tool side relative to $\{\mathrm{S}\} . \theta$ and $\theta_{0}$ denotes the variable and the initial angle of the revolute joint $\mathrm{A}$, respectively. $r_{m}$ and $r_{t}$ are effective diameter of the joints on the mounting side and the tool side. $l$ denotes the length of the leaf spring.

As to the motion of the single leaf spring, the pose of the spring's tip frame, with respect to the local frame on the distal end of the spring $\{L\}$, can be derived as

$$
\boldsymbol{g}_{l t}=\boldsymbol{g}_{s l}^{-1} \boldsymbol{g}_{\mathrm{s} t},
$$

where $\boldsymbol{g}_{s l} \in S E$ (3) denotes the pose of the local frame on the distal end of the spring with respect to $\{S\}$ and $\boldsymbol{g}_{s t} \in S E(3)$ relates to the pose of the spring's tip frame, with respect to $\{S\}$, which can be calculated from $x, y, \phi$ and the known parameters.

\subsection{Kinetostatics}

On the basis of our prior work [26, 27], the leaf springs in the elastic limbs are discretized into a number of small segments, as illustrated in Figure 6. For each segment, a six-DOF linkage consisting of rigid bodies and elastic joints can be attained based on the principal axes decomposition of the structural compliance matrix [41]. Then, the force-deflection behavior of the discretized elastic segments can be approximated by the kinetostatics of the equivalent linkages. Connecting all the segments one after another, a hyper-redundant rigid-body mechanism with elastic joints can be constructed to represent the large deflection problems of the leaf springs.

Due to its thin-walled structure, the thickness of the leaf spring is much smaller than its width. It's appropriate to regard the leaf spring as totally stiff in the direction perpendicular to the narrow edge. On the other hand, the effects of shearing and compression are neglectable compared with the bending one [42]. Thus, only the revolute

$$
\left\{\begin{aligned}
\boldsymbol{g}_{l t} & =\prod_{i=1}^{2 n} \exp \left(\hat{\xi}_{i} \theta_{i}\right) \boldsymbol{g}_{l t, 0}, \\
\boldsymbol{\tau} & =\boldsymbol{K}_{\theta} \boldsymbol{\theta}-\boldsymbol{J}_{\theta}^{\mathrm{T}} \boldsymbol{F} \rightarrow \mathbf{0},
\end{aligned}\right.
$$

where $\boldsymbol{\theta}_{i}=\left[\theta_{1}, \ldots, \theta_{2 n}\right]^{\mathrm{T}} \in \mathbb{R}^{2 n \times 1}$ denotes the joint variables of the whole spring. $\xi_{i}=\operatorname{Ad}\left(\boldsymbol{g}_{0,0}, \ldots, \boldsymbol{g}_{i-1,0}\right) \boldsymbol{t}_{i}$ are the joint twists, transformed from their local frames to $\{L\} ; \boldsymbol{t}_{i}$ denote the joint twists of the segments in their local frames. $\boldsymbol{g}_{l t, 0}=\boldsymbol{g}_{0,0} \boldsymbol{g}_{1,0} \cdots \boldsymbol{g}_{2 n, 0}$ relates to the initial pose of the leaf spring with respect to $\{L\} .2 n$ is the total number of joints.

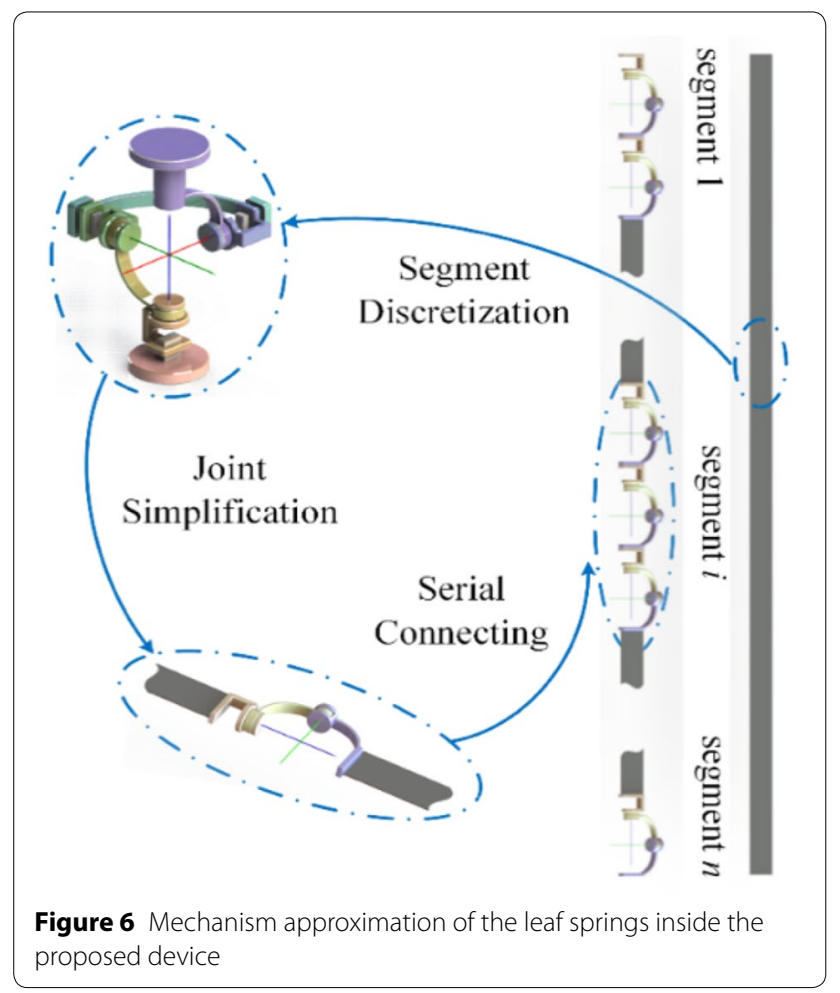


$\boldsymbol{K}_{\theta}=\operatorname{diag}\left(1 / c_{1,1}, 1 / c_{1,2}, 1 / c_{2,1}, 1 / c_{2,2}, \cdots, 1 / c_{n, 1}, 1 / c_{n, 2}\right)$ denotes the overall joint stiffness matrix, where $c_{i, 1}=\delta / E I_{x x}, c_{i, 2}=\delta / G I_{z z}$ denote the compliance of the approximated joints for bending and torsion. $\delta, E, G, I_{x x}$ and $I_{z z}$ are the length of each small segment, the elastic modulus, the shear modulus, and the moment of inertia on bending and torsion direction, respectively. And $\boldsymbol{F}$ corresponds to the external wrench exerted at the tip. Here, the Jacobian matrix $\boldsymbol{J}_{\theta}$ can be derived as

$$
\boldsymbol{J}_{\theta}=\left(\frac{\partial \dot{\boldsymbol{g}}_{s t}}{\partial \boldsymbol{\theta}} \boldsymbol{g}_{s t}^{-1}\right)^{\vee}=\left[\xi_{1}^{\prime}, \xi_{2}^{\prime}, \cdots, \xi_{2 n}^{\prime}\right] \in \mathbb{R}^{6 \times 2 n}
$$

where $\xi^{\prime}{ }_{k}=\operatorname{Ad}\left(\exp \left(\hat{\xi}_{1} \theta_{1}\right) \cdots \exp \left(\hat{\xi}_{k-1} \theta_{k-1}\right)\right) \xi_{k}$ relate to the joint twists in the current configuration and are represented with respect to $\{\mathrm{L}\}$.

Then, an unconstrained optimization model is formulated to accomplish the force-deflection problems of the corresponding hyper-redundant mechanism, which can be defined as

$$
\min \boldsymbol{c}(\boldsymbol{x})=\left[\begin{array}{r}
\log \left(\boldsymbol{g}_{t}^{-1} \boldsymbol{g}_{l t}(\boldsymbol{\theta})\right)^{\vee} \\
\boldsymbol{K}_{\theta} \boldsymbol{\theta}-\boldsymbol{J}_{\theta}^{\mathrm{T}} \boldsymbol{F}
\end{array}\right],
$$

where $\boldsymbol{x}=[\boldsymbol{\theta}, \boldsymbol{F}]^{\mathrm{T}} \in \mathbb{R}^{(2 n+7) \times 1}$ denotes the variables of the optimization problem. $\boldsymbol{g}_{t} \in S E(3)$ denotes the target pose for the tip-frame of each leaf spring. $\log \left(\boldsymbol{g}_{t}^{-1} \boldsymbol{g}_{l t}(\boldsymbol{\theta})\right)^{\vee} \in \mathbb{R}^{6 \times 1}$ corresponds to the twist deviation of current pose from the target one.

Thus, the gradient of the objective function Eq. (7) can be written as

$$
\nabla=\left[\frac{\partial \boldsymbol{c}}{\partial \boldsymbol{\theta}}, \frac{\partial \boldsymbol{c}}{\partial \boldsymbol{F}}\right]=\left[\begin{array}{cc}
\boldsymbol{J}_{\theta} & \mathbf{0} \\
\boldsymbol{K}_{\theta}+\boldsymbol{K}_{\boldsymbol{J}} & -\boldsymbol{J}_{\theta}^{\mathrm{T}}
\end{array}\right]
$$

where $K_{\boldsymbol{J}} \in \mathbb{R}^{2 n \times 2 n}$ is a configuration-dependent stiffness item. Please refer to Ref. [26] for more details.

Then, the update theme for the variables in this hybrid equilibrium problem can be represented as

$$
\boldsymbol{x}^{j+1}=\boldsymbol{x}^{j}+\nabla^{-1} \boldsymbol{c}^{j}
$$

which will be iteratively repeated until the objective function $\boldsymbol{c}$ approaches zero and the variable $\boldsymbol{x}$ converges stably. As a result, the rotations of all the approximated joints and the generated force in the equilibrium configuration can be simultaneously obtained in terms of the resultant $\boldsymbol{\theta}$ and $\boldsymbol{F}$.

\subsection{Compliance Analysis}

In this section, the compliance of the proposed device under the compliant status is analyzed. The geometric parameters of the analyzed device and the mechanical properties of the leaf spring are given in Table 1. It is
Table 1 Lengths and mechanical properties of the leaf springs

\begin{tabular}{llll}
\hline Parameter & $\boldsymbol{I}(\mathbf{m})$ & $\boldsymbol{r}_{\boldsymbol{t}}(\mathbf{m})$ & $\boldsymbol{r}_{\boldsymbol{m}}(\mathbf{m})$ \\
\hline Value & 0.07 & 0.08 & 0.05 \\
Parameter & $h_{W}(\mathrm{~m})$ & $h_{T}(\mathrm{~m})$ & $E(\mathrm{GPa})$ \\
Value & $5 \times 10^{-3}$ & $2 \times 10^{-4}$ & 67 \\
Parameter & $\mu$ & $I_{x x}\left(\mathrm{~m}^{4}\right)$ & $I_{z z}\left(\mathrm{~m}^{4}\right)$ \\
Value & 0.3 & $3.3 \times 10^{-15}$ & $1.3 \times 10^{-14}$ \\
\hline
\end{tabular}

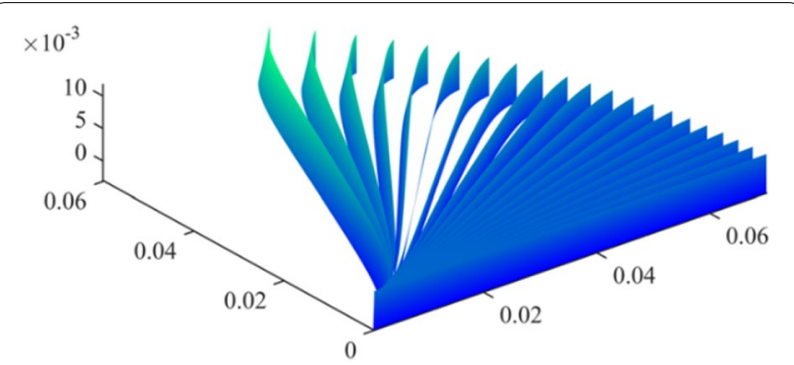

Figure 7 Deformations of the single leaf spring from 0 to $60 \mathrm{~mm}$

worth noting that the device is central symmetric, and lengths of all the leaf springs are same. $h_{W}$ and $h_{T}$ denotes the width and thickness of the leaf spring, respectively. $\mu$ is Poisson's ratio of the springs.

Based on the above analysis, the horizontal displacement of the device's mounting side is set from 0 to 60 $\mathrm{mm}$. Then, the deflection of the leaf spring is calculated every $3 \mathrm{~mm}$ and is illustrated in Figure 7 . At the same time, Figure 8 depicts the variation of the generated force during the passive motion. According to the figures, it is apparent that the leaf spring is easy to be deformed and can not generate great force. The generated force has an upper boundary less than $10.5 \mathrm{~N}$ and the force decreases as the tool side keep moving after passing the boundary points.

Considering the relatively low generated force, the device is proved to bring sufficient compliance to the manipulator or the operator when misalignment happens in applications like assembly automation or human-robot interaction. Benefiting from the upper boundary, the device will ensure the protection regardless of the positioning error. Moreover, the passive compliance is attained from the inherent structure and no sensors or complicated control algorithms is needed during the manipulation. As the same time, the device will act compliant immediately however fast the misalignment or the collision occurs, which is hard to realize by the active compliance because of its limited bandwidth [44]. 


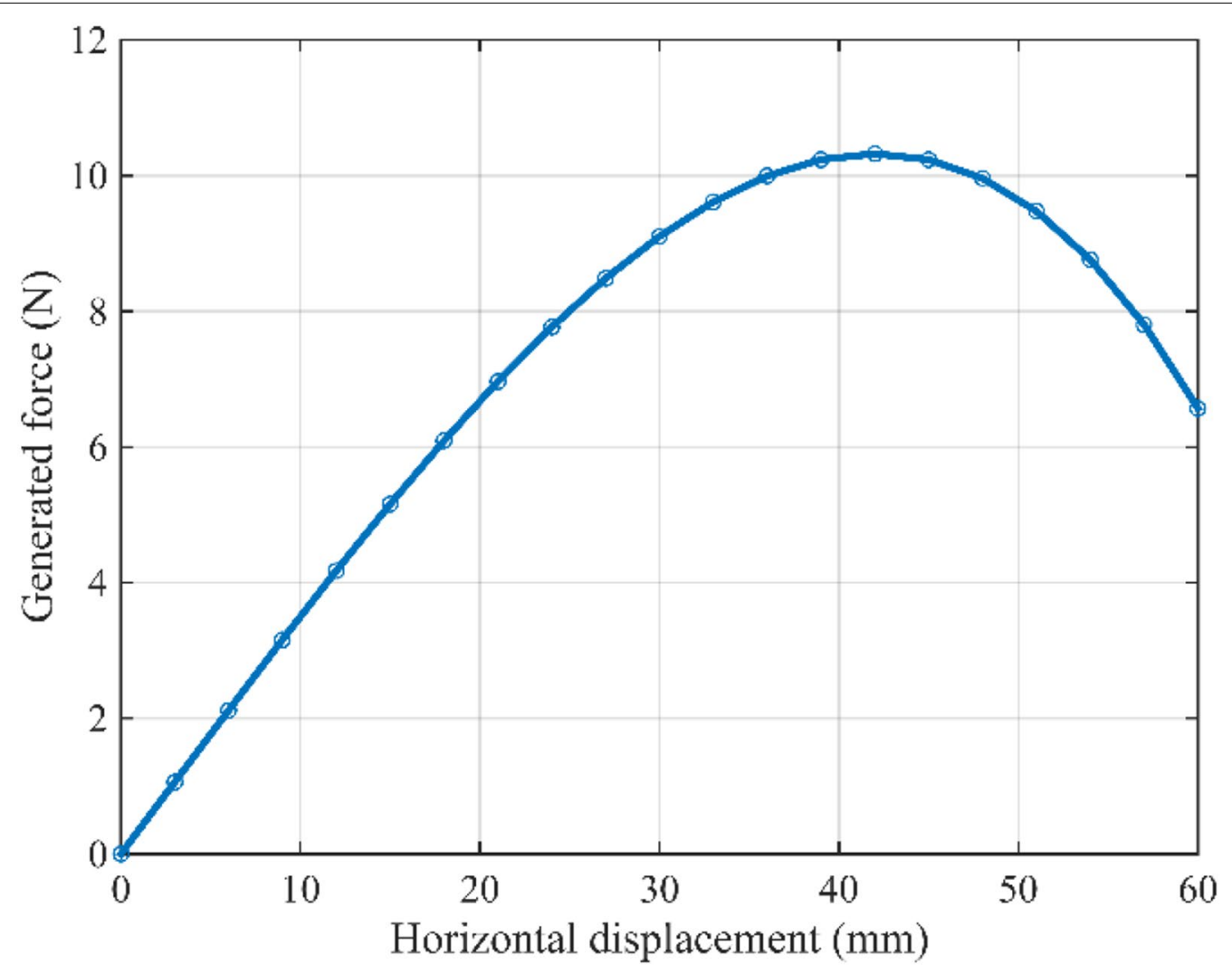

Figure 8 Generated forces of the single leaf spring from 0 to $60 \mathrm{~mm}$

\section{Experiments}

To validate the capability of the stiffness changing of the proposed device, several experiments were conducted on the prototype with the geometric parameters shown in Table 1 . The prototype was fixed upon the end effector of a six-DOF industrial robot (UR 10) from which the motion generated during the experiments. The motion of the tool side of the prototype was measured by a motion capture system (OptiTrack Prime 41) with the absolute measurement accuracy around $0.3 \mathrm{~mm}$.

The first experiment aimed to show the stiffness of the prototype under different statuses. As shown in Figure 9, the tool side was imposed to knock into a fixed barrier under the actuation of the industrial robot; the prototype was set at the stiff and the compliant status, respectively. A six-axis force-torque sensor (ATI Mini 45) was mounted between the barrier and a fixed platform to measure the interaction force. Then, different displacements were imposed at the mounting side of the prototype while the tool side kept motionless due to the interaction with the fixed barrier.

Figure 10 illustrates the correlations between the generated forces and the relative displacements of the prototype under two different statuses. It is apparent that

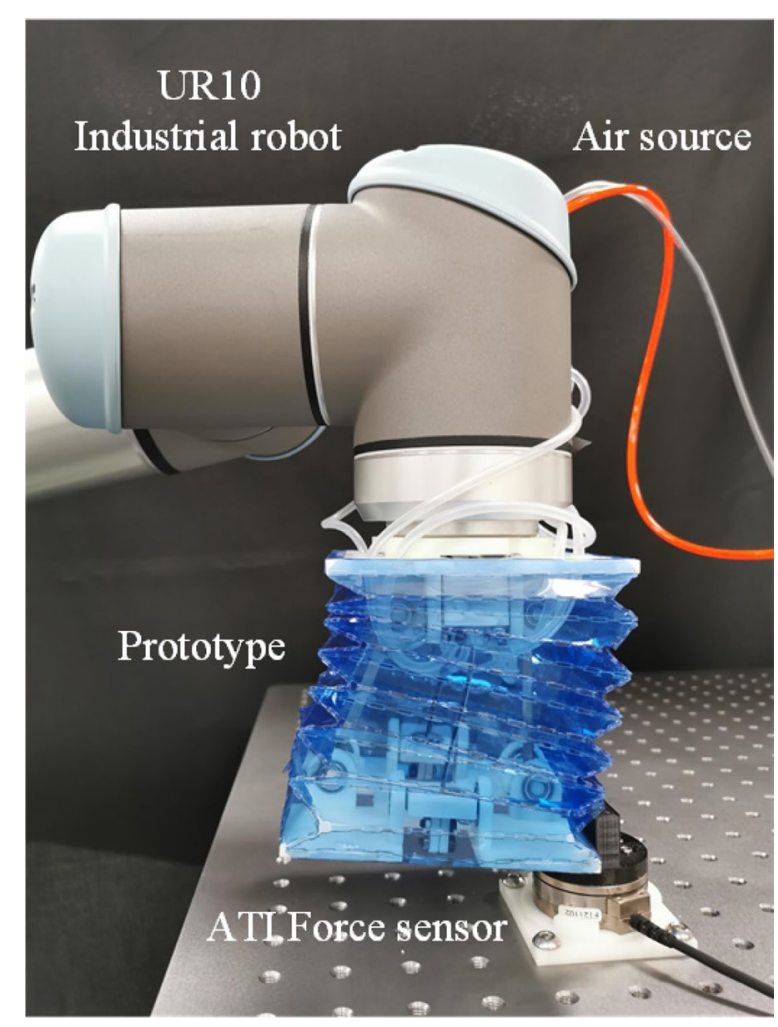

Figure 9 Setup of the stiffness tests 


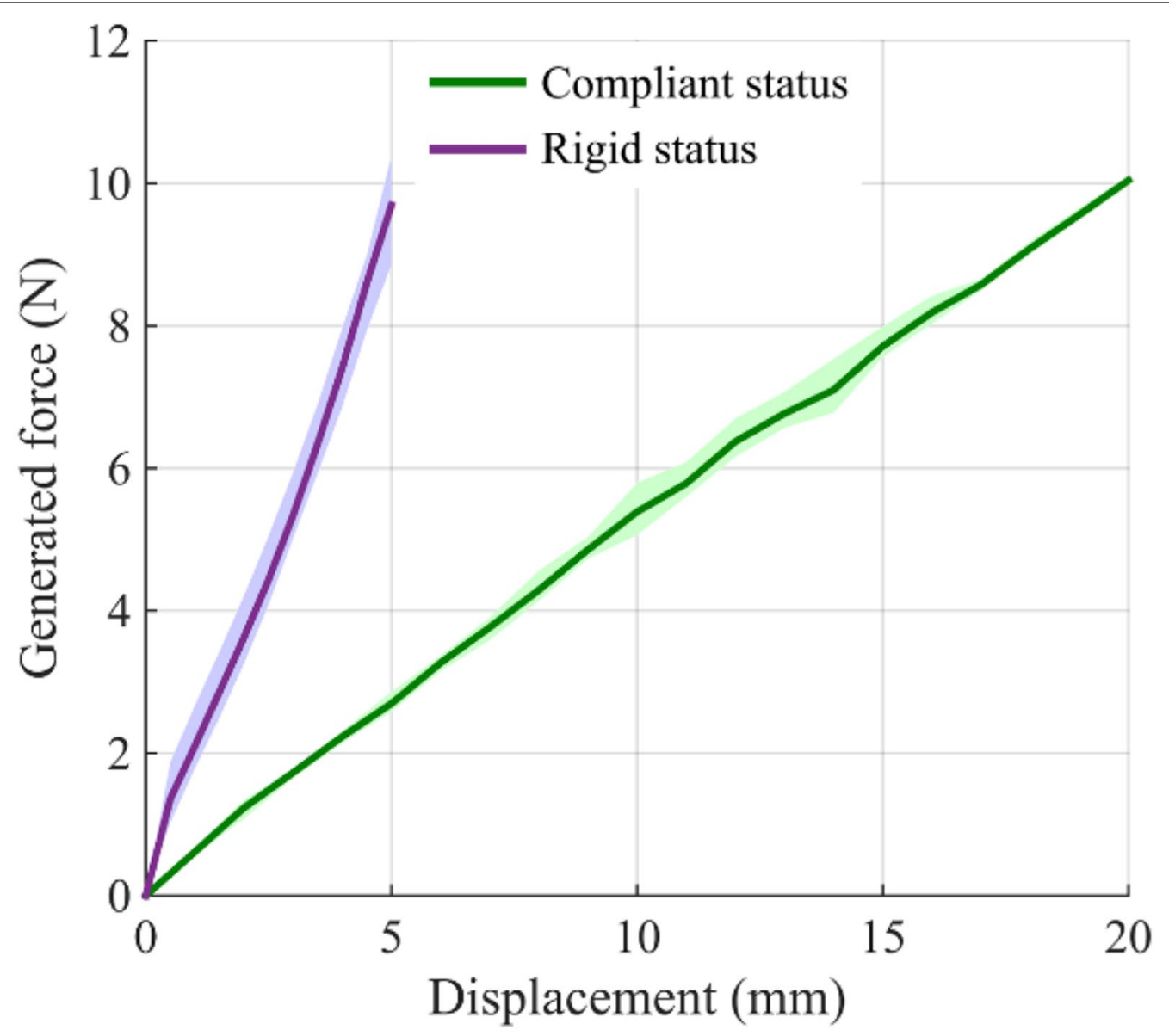

Figure 10 Correlations between the generated forces and the displacements under two statuses

the prototype showed high stiffness under the stiff status. The generated force rose up to $10 \mathrm{~N}$ when the relative displacement between the two sides was just around $5 \mathrm{~mm}$. It is worth noting that the relative displacement in this test was mainly generated from the assembly gap and the structural deformation of the device. The stiffness should be much more enlarged through replacing the plastic parts by the metal ones. Hence, the displacement was controlled less than $5 \mathrm{~mm}$ to prevent the plastic three-dimensional printed elements from damage. On the contrary, the displacement could be much larger in the test under the compliant status. The reconfigurable elastic inner skeleton as well as the origami chamber was easily deformed to adapt the relative movement between the mounting side and the tool side. The compliance under such status was noticeable and the generated force was around $10 \mathrm{~N}$ when the displacement reached $20 \mathrm{~mm}$. It should be noted that the measured force was generated from two deflected leaf springs, so that the measured force was almost twice the predicted one.
In the second experiment, the response of the stiffness variation was tested. Similar to the first experiment, the tool side was first imposed to knock into the barrier fixed upon the six-axis force-torque sensor under the stiff status. Then, the status was controlled to be switched under the actuation of the Si-Mo pneumatic actuation system. The interaction force during such process was record by the force sensor and Figure 11 illustrates the response of the prototype. It is apparent that the stiffness of the prototype can be changed rapidly with a reaction time around $80 \mathrm{~ms}$ from the stiff status to the compliant one. As a consequence, the proposed device was proved to have the capability of switching its stiffness in a fast, simple and straightforward manner.

The last experiment was designed to prove the torsional strength augment from the origami shell, which has an apparent influence on the behavior of the device under the compliant status. As shown in Figure 12(a), the torsion was manually imposed on the tool side with a sixaxis force-torque sensor mounted between the tool side 


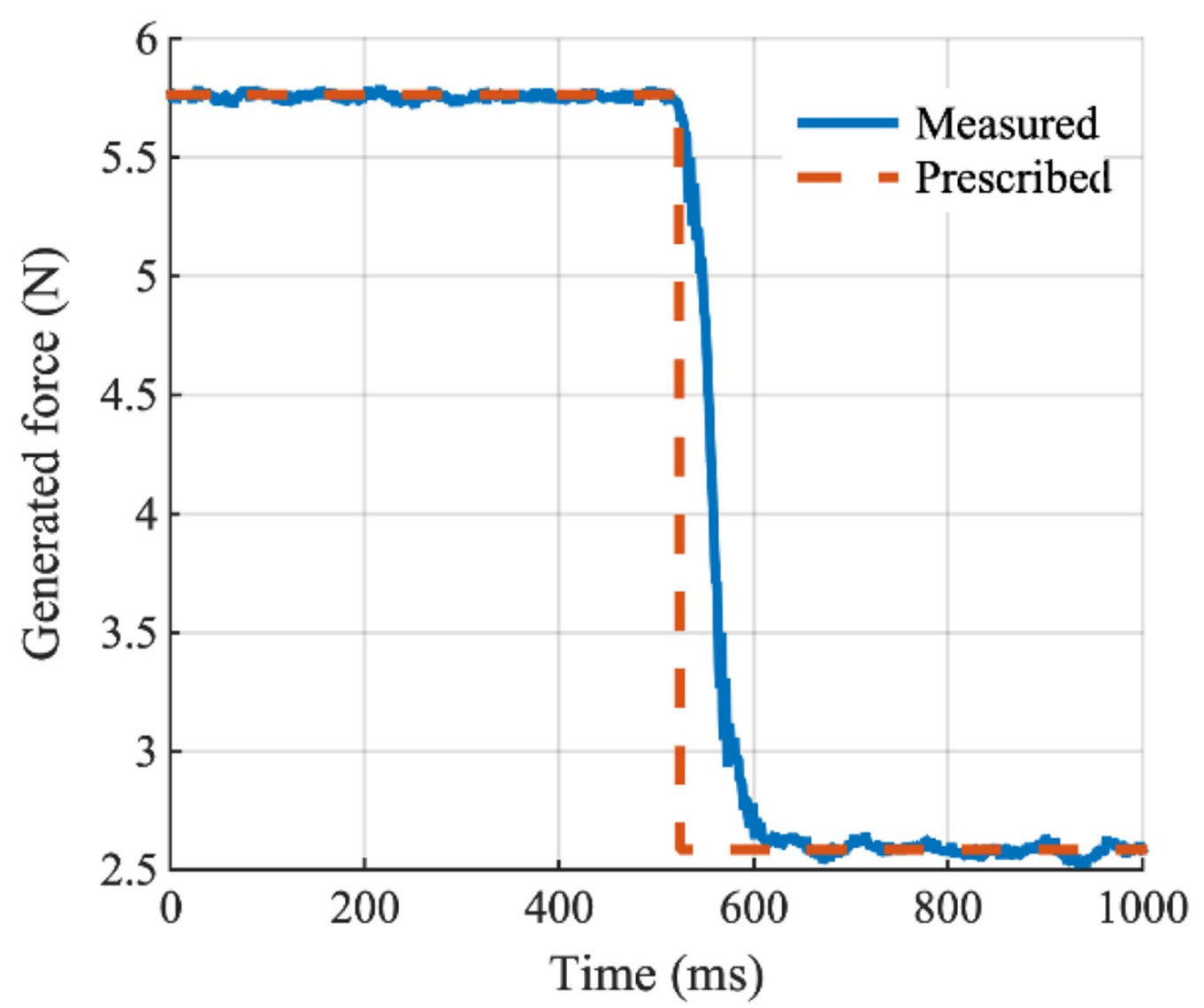

Figure 11 Response of the stiffness variation

and the rotation bar. Both the device with and without the origami shell were tested. As shown in Figure 12(b), the tool side rotated more than $16^{\circ}$ under the torsional force around $0.3 \mathrm{~N} \cdot \mathrm{m}$ without the origami shell. However, almost no rotation generated on the prototype with the origami shell under the same torsional forces. It was apparent that the device without the origami shell was easy to be twisted while the device with the origami shell seemed stable. Hence, the torsional strength of the proposed device was proved to be significantly enhanced by the origami shell.

\section{Conclusions}

In this paper, a novel stiffness variable passive compliance device that consists of a reconfigurable elastic inner skeleton, an origami shell and a Si-Mo pneumatic actuation system is proposed. Controlling the self-rotation of the leaf springs, the arrangement of the elastic links and the passive joints can be changed and the stiffness variation of the device is as thus realized. The device can be used for precise positioning or applications with high acceleration /deceleration under the stiff status and providing passive compliance or protection under the compliant status. The Si-Mo pneumatic actuation system can switch the stiffness of the device in a fast, simple and straightforward way and the device has potential applications in some special environments as no electrical elements needs to be mounted on the device.

The kinetostatics as well as the compliance of the device is analyzed based on an efficient approach to large deflection problems. A prototype has been built to assess the proposed concept. The experimental results show that the device possesses relatively low stiffness under the compliant status and high stiffness under the stiff status with a status switching speed around $80 \mathrm{~ms}$. The device generates only $10 \mathrm{~N}$ when the relative displacement between the two sides is around $20 \mathrm{~mm}$ under the compliant status. The laser-cut origami shell significantly enhances the torsional strength of the device and the interaction safety can benefit from its inherent soft structure. 

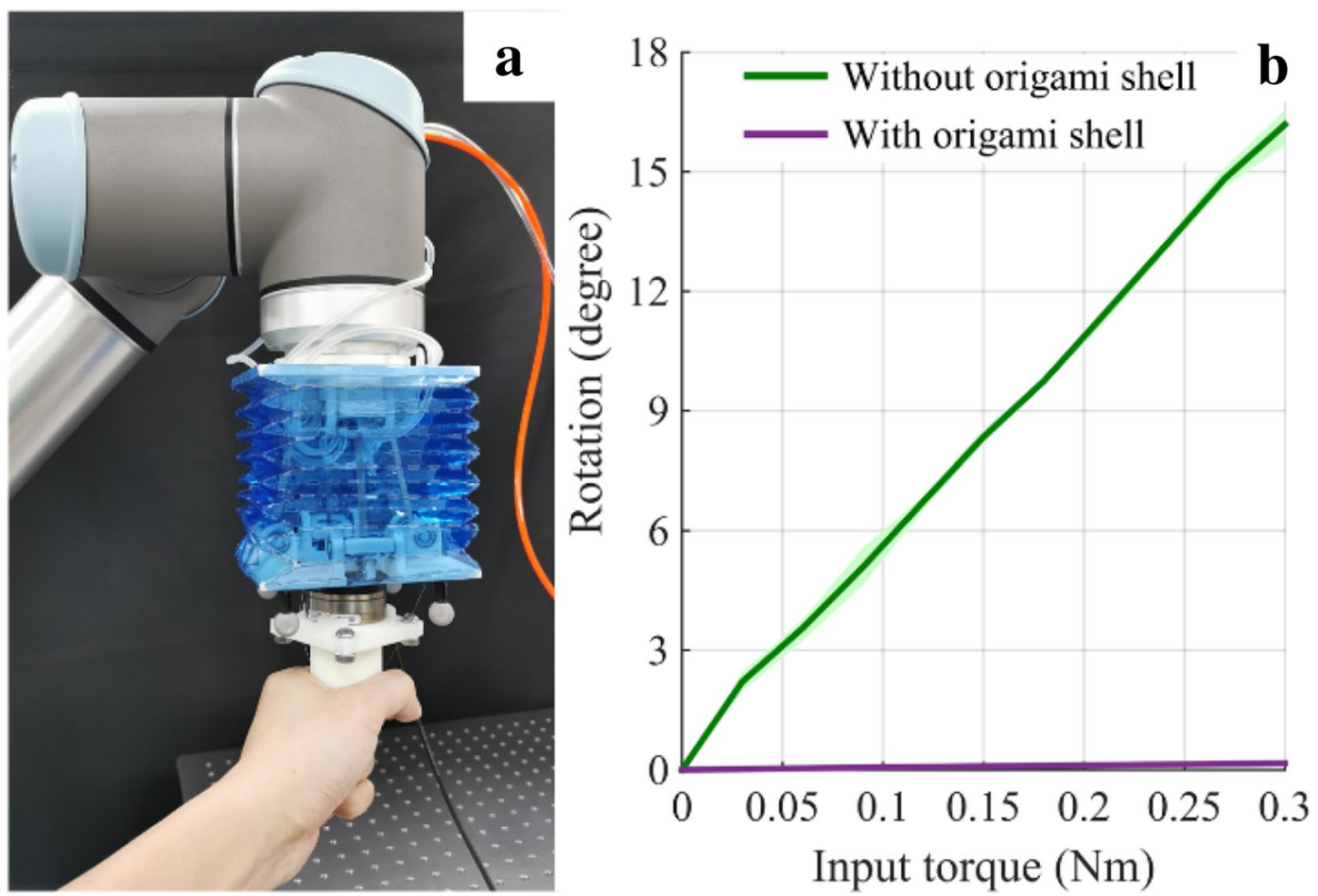

Figure 12 Experiment and results of the torsional strength tests with/without the origami shell

\section{Acknowledgements}

Not applicable.

\section{Authors' Contributions}

$\mathrm{ZZ}, \mathrm{GC}$ and HW were in charge of the whole trial; ZZ and GC wrote the manuscript; WF and YW assisted with prototyping and experiments; LK assisted with structural design and modeling. All authors read and approved the final manuscript

\section{Authors' Information}

Zhuang Zhang, born in 1993, is currently a PhD candidate at Shanghai Key Laboratory of Digital Manufacture for Thin-walled Structures, Shanghai Jiao Tong University, China. His research interests include mechanisms and robotics, soft actuators and flexible manipulators.

Genliang Chen, born in 1982, is currently an associate professor at Shanghai Key Laboratory of Digital Manufacture for Thin-walled Structures, and State Key Laboratory of Mechanical System and Vibration, Shanghai Jiao Tong University, China. His research interests include mechanisms and robotics, flexible manipulators, manipulator calibration and assembly automation.

Weicheng Fan, born in 1997, is currently a master candidate at Shanghai Key Laboratory of Digital Manufacture for Thin-walled Structures, Shanghai Jiao Tong University, China.

Wei Yan, born in 1998, is currently a master candidate at Shanghai Key Laboratory of Digital Manufacture for Thin-walled Structures, Shanghai Jiao Tong University, China.

Lingyu Kong, born in 1988, is currently a researcher at Intelligent Robot Research Center, Zhejiang Lab, Hangzhou, China. He received his PhD degree from Shanghai Jiao Tong University, China in 2018. His research interests include mechatronics engineering, robotics and manipulator calibration.

Hao Wang, born in 1973, is currently a professor at Shanghai Key Laboratory of Digital Manufacture for Thin-walled Structures, and State Key Laboratory of Mechanical System and Vibration, Shanghai Jiao Tong University, China. He serves as the Vice Dean of School of Mechanical Engineering, Shanghai Jiao Tong University, China. His research interests include mechanisms and robotics, assembly automation, multibody system dynamics, and intelligent manufacturing.

\section{Funding}

Supported in part by National Key Research and Development Program of China (Grant No. 2017YFE0111300), National Natural Science Foundation of China (Grant No. 51875334), and State Key Lab of Digital Manufacturing Equipment and Technology (Huazhong University of Science and Technology) (Grant No. DMETKF2019007).

\section{Competing Interests}

The authors declare no competing financial interests.

\section{Author Details}

${ }^{1}$ Shanghai Key Laboratory of Digital Manufacture for Thin-walled Structures, Shanghai Jiao Tong University, Shanghai 200240, China. ${ }^{2}$ State Key Laboratory of Mechanical System and Vibration, Shanghai Jiao Tong University, Shanghai 200240, China. ${ }^{3}$ Intelligent Robot Research Center, Zhejiang Lab, Hangzhou 311100, China.

Received: 31 January 2020 Revised: 3 September 2020 Accepted: 7 October 2020

Published online: 02 November 2020

\section{References}

[1] A De Santis, B Siciliano, A De Luca, et al. An atlas of physical human-robot interaction. Mechanism and Machine Theory, 2008, 43(3): 253-270.

[2] D E Whitney. Historical perspective and state of the art in robot force control. The International Journal of Robotics Research, 1987, 6(1): 3-14.

[3] S Haddadin, A Albu-Schäffer, G Hirzinger. Requirements for safe robots: Measurements, analysis and new insights. The International Journal of Robotics Research, 2009, 28(11-12): 1507-1527.

[4] W Wang, R N K Loh, EY Gu. Passive compliance versus active compliance in robot-based automated assembly systems. Industrial Robot: An International Journal, 1998, 25(1): 48-57.

[5] N Hogan, S P Buerger. Robotics and Automation Handbook. New York: CRC Press, 2004: 375-398. 
[6] TLefebvre, J Xiao, H Bruyninckx, et al. Active compliant motion: A survey. Advanced Robotics, 2005, 19(5): 479-499.

[7] K Goris, J Saldien, B Vanderborght, et al. How to achieve the huggable behavior of the social robot Probo? A reflection on the actuators. Mechatronics, 2011, 21(3): 490-500.

[8] RVan Ham, T G Sugar, B Vanderborght, et al. Compliant actuator designs. IEEE Robotics \& Automation Magazine, 2009, 16(3): 81-94.

[9] J J Park, J B Song. A nonlinear stiffness safe joint mechanism design for human robot interaction. Journal of Mechanical Design, 2010, 132(6): 061005.

[10] D E Whitney, J M Rourke. Mechanical behavior and design equations for elastomer shear pad remote center compliances. Journal of Dynamic Systems, Measurement, and Control, 1986, 108(3): 223-232.

[11] Š Havlík. A new elastic structure for a compliant robot wrist. Robotica, 1983, 1(2): 95-102.

[12] N Ciblak, H Lipkin. Design and analysis of remote center of compliance structures. Journal of Robotic Systems, 2003, 20(8): 415-427.

[13] S Lee. Development of a new variable remote center compliance (VRCC) with modified elastomer shear pad (ESP) for robot assembly. IEEE Transactions on Automation Science and Engineering, 2005, 2(2): 193-197.

[14] J Yu, Y Zhao, G Chen, et al. Realizing controllable physical interaction based on an electromagnetic variable stiffness joint. Journal of Mechanisms and Robotics, 2019, 11(5): 054501.

[15] P H Kuo, A D Deshpande. A novel joint design for robotic hands with humanlike nonlinear compliance. Journal of Mechanisms and Robotics, 2016, 8(2).

[16] D Shin, I Sardellitti, Y L Park, et al. Design and control of a bio-inspired human-friendly robot. The International Journal of Robotics Research, 2010, 29(5): 571-584.

[17] TW Seo, M Sitti. Tank-like module-based climbing robot using passive compliant joints. IEEE/ASME Transactions on Mechatronics, 2012, 18(1): 397-408.

[18] J Choi, S Hong, W Lee, et al. A robot joint with variable stiffness using leaf springs. IEEE Transactions on Robotics, 2011, 27(2): 229-238.

[19] M Zhu, Y Mori, TWakayama, et al. A fully multi-material three-dimensional printed soft gripper with variable stiffness for robust grasping. Soft Robotics, 2019, 6(4): 507-519.

[20] S Kawamura, TYamamoto, D Ishida, et al. Development of passive elements with variable mechanical impedance for wearable robots. Proceedings 2002 IEEE International Conference on Robotics and Automation, IEEE, 2002, 1: 248-253.

[21] A Bamotra, P Walia, A V Prituja, et al. Layer-jamming suction grippers with variable stiffness. Journal of Mechanisms and Robotics, 2019, 11(3): 035003.

[22] TWang, J Zhang, Y Li, et al. Electrostatic layer jamming variable stiffness for soft robotics. IEEE/ASME Transactions on Mechatronics, 2019, 24(2): 424-433.

[23] Y J Kim, S Cheng, S Kim, et al. A novel layer jamming mechanism with tunable stiffness capability for minimally invasive surgery. IEEE Transactions on Robotics, 2013, 29(4): 1031-1042.

[24] Y Tao, TWang, Y Wang, et al. A new variable stiffness robot joint. Industrial Robot: An International Journal, 2015, 42(4): 371-378.
[25] S Bi, C Liu, H Zhao, et al. Design and analysis of a novel variable stiffness actuator based on parallel-assembled-folded serial leaf springs. Advanced Robotics, 2017, 31(18): 990-1001.

[26] G Chen, Z Zhang, H Wang. A general approach to the large deflection problems of spatial flexible rods using principal axes decomposition of compliance matrices. Journal of Mechanisms and Robotics, 2018, 10(3): 031012.

[27] G Chen, Z Zhang, L Kong, et al. Analysis and validation of a flexible planar two degrees-of-freedom parallel manipulator with structural passive compliance. Journal of Mechanisms and Robotics, 2020, 12(1): 011011.

[28] J S Dai, M Zoppi, X Kong. Advances in reconfigurable mechanisms and robots I. London: Springer, 2012.

[29] G Wei, J S Dai, S Wang, et al. Kinematic analysis and prototype of a metamorphic anthropomorphic hand with a reconfigurable palm. International Journal of Humanoid Robotics, 2011, 8(3): 459-479.

[30] G Wei, J S Dai. Advances in robot kinematics. Cham: Springer, 2014.

[31] Y Yoshimura. On the mechanism of buckling of a circular cylindrical shell under axial compression. Reports of the Institute of Science and Technology of the University of Tokyo, 1955

[32] D Rus, C Sung. Spotlight on origami robots. Science Robotics, 2018, 3(15): eaat0938.

[33] G Wei, J S Dai. Origami-inspired integrated planar-spherical overconstrained mechanisms. Journal of Mechanical Design, 2014, 136(5): 051003.

[34] Y Chen, W Lv, R Peng, et al. Mobile assemblies of four-spherical-4R-integrated linkages and the associated four-crease-integrated rigid origami patterns. Mechanism and Machine Theory, 2019, 142: 103613.

[35] Z Zhang, G Chen, HWu, et al. A pneumatic/cable-driven hybrid linear actuator with combined structure of origami chambers and deployable mechanism. IEEE Robotics and Automation Letters, 2020, 5(2): 3564-3571.

[36] L Paez, G Agarwal, J Paik. Design and analysis of a soft pneumatic actuator with origami shell reinforcement. Soft Robotics, 2016, 3(3): 109-119.

[37] R V Martinez, C R Fish, X Chen, et al. Elastomeric origami: programmable paper-elastomer composites as pneumatic actuators. Advanced Functional Materials, 2012, 22(7): 1376-1384.

[38] M Luo, R Yan, Z Wan, et al. OriSnake: Design, fabrication, and experimental analysis of a 3-D origami snake robot. IEEE Robotics and Automation Letters, 2018, 3(3): 1993-1999.

[39] J Santoso, E H Skorina, M Luo, et al. Design and analysis of an origami continuum manipulation module with torsional strength. 2017 IEEE/ RSJ International Conference on Intelligent Robots and Systems (IROS), IEEE, 2017: 2098-2104.

[40] K Miura, T Tachi. Synthesis of rigid-foldable cylindrical polyhedra. Symmetry: Art and Science, 2010: 204-213.

[41] G Chen, H Wang, Z Lin, et al. The principal axes decomposition of spatial stiffness matrices. IEEE Transactions on Robotics, 2015, 31(1): 191-207.

[42] L Howell. Compliant mechanisms. John Wiley \& Sons, 2001.

[43] G Chen, H Wang, Z Lin. Determination of the identifiable parameters in robot calibration based on the POE formula. IEEE Transactions on Robotics, 2014, 30(5): 1066-1077.

[44] A Bicchi, G Tonietti. Fast and" soft-arm" tactics [robot arm design]. IEEE Robotics \& Automation Magazine, 2004, 11(2): 22-33.

\section{Submit your manuscript to a SpringerOpen ${ }^{\circ}$ journal and benefit from:}

- Convenient online submission

- Rigorous peer review

- Open access: articles freely available online

- High visibility within the field

- Retaining the copyright to your article

Submit your next manuscript at springeropen.com 